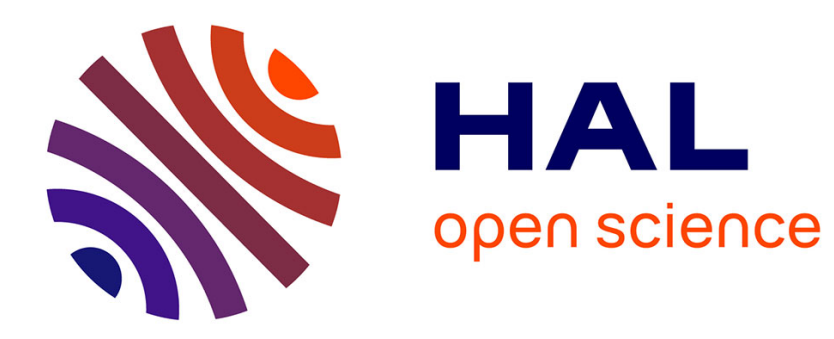

\title{
Photoconsommation d'oxygène chez Zea mays L
}

Pascale Jolivet-Tournier, Richard Gerster

\section{To cite this version:}

Pascale Jolivet-Tournier, Richard Gerster. Photoconsommation d'oxygène chez Zea mays L. Agronomie, 1983, 3 (9), pp.897-902. hal-00884587

\section{HAL Id: hal-00884587 https://hal.science/hal-00884587}

Submitted on 1 Jan 1983

HAL is a multi-disciplinary open access archive for the deposit and dissemination of scientific research documents, whether they are published or not. The documents may come from teaching and research institutions in France or abroad, or from public or private research centers.
L'archive ouverte pluridisciplinaire HAL, est destinée au dépôt et à la diffusion de documents scientifiques de niveau recherche, publiés ou non, émanant des établissements d'enseignement et de recherche français ou étrangers, des laboratoires publics ou privés. 


\title{
Photoconsommation d'oxygène chez Zea mays L.
}

\author{
Pascale JOLIVET-TOURNIER \& Richard GERSTER (*)
}

I.N.R.A., I.N.A.-P.G., Laboratoire de Chimie biologique et de Photophysiologie, F 78850 Thiverval-Grignon (*) C.E.N. Cadarache, Département de Biologie, Service de Radioagronomie, B.P. 1, F 13115 Saint-Paul-lezDurance

\section{RÉSUMÉ}

A l'aide de l'isotope stable ${ }^{18} \mathrm{O}$, il a été possible de mesurer une photoconsommation d'oxygène au point de compensation en $\mathrm{CO}_{2}$ chez le mais. Comme chez les végétaux de type $\mathrm{C}_{3}$, cette prise d'oxygène augmente en même temps que la concentration en $\mathrm{O}_{2}$; par contre, elle n'est fortement diminuéc que lorsque la teneur en $\mathrm{CO}$, est très élevée $(2$ à 4 p. 100). Elle varie également en fonction du stade de développement du végétal. Ces résultats suggèrent l'existence d'un métabolisme photorespiratoire chez le maïs.

Mots clés additionnels: Maïs, ${ }^{18} \mathrm{O}$, point de compensation en $\mathrm{CO}_{2}$, concentration en $\mathrm{O}_{2}$, stade de développement.

${ }^{18} \mathrm{O}_{2}$ was used to determine $\mathrm{O}_{2}$-uptake by maize leaves. In this plant, light-dependent $\mathrm{O}_{2}$-uptake was not negligible at the $\mathrm{CO}_{2}$ compensation point. As for $\mathrm{C}_{3}$ plants, $\mathrm{O}_{2}$-uptake increased with $\mathrm{O}_{2}$ concentration ; it was strongly limited only by high $\mathrm{CO}_{2}$ concentrations ( 2 to $4 \%$ ). The rate of uptake was also dependent on the stage of plant development. These results suggest that photorespiration is operating in maize leaves.

Additional key words : Maize, ${ }^{18} \mathrm{O}, \mathrm{CO}_{2}$ compensation point, $\mathrm{O}_{2}$ concentration, stage of development.

\section{INTRODUCTION}

La photorespiration au plan des échanges gazeux se traduit par une consommation d'oxygène et une production de gaz carbonique simultanément à la production d'oxygène et la consommation de gaz carbonique de la photosynthèse. Ce phénomène est intense chez les plantes de type métabolique $\mathrm{C}_{3}$ et entraîne une diminution de la productivité végétale. Les différentes méthodes utilisées pour mettre en évidence le dégagement photorespiratoire de gaz carbonique chez le maïs et, de manière plus générale, chez les végétaux de type $C_{4}$ se sont révélées souvent inefficaces. Le maïs ne manifeste pas de "pic respiratoire "typique lors de la transition lumière-obscurité même en atmosphère d'oxygène pur (FORRESTER et al., 1966). Il ne présente pas de dégagement de $\mathrm{CO}_{2}$ dans un air dépourvu de ce gaz ( $\mathrm{EL}$ SHARKAWY et al., 1967). Lorsque le végétal est maintenu dans une enceinte en présence de ${ }^{14} \mathrm{CO}_{2}$, aucune dilution isotopique du ${ }^{14} \mathrm{CO}_{2}$ externe par du ${ }^{12} \mathrm{CO}_{2}$ d'origine photorespiratoire n'est constatée (HEw et al., 1969). Enfin, le point de compensation en $\mathrm{CO}_{2}$, concentration pour laquelle la photosynthèse brute est exactement compensée par le dégagement de gaz carbonique, est généralement inférieur à $10 \mu \mathrm{l} \cdot \mathrm{l}^{-1}$ dans le cas du maïs (FORRESTER et al., 1966 ;
ZELITCH, 1971). En ce qui concerne l'étude de l'inhibition de la photosynthèse du mais par $\mathrm{O}_{2}$ ou " effet WARBURG ", les résultats obtenus conduisent à des conclusions contradictoires VOSKRESENSKAYA et al. (1974) constatent que la photosynthèse apparente des feuilles est insensible aux changements de la concentration en $\mathrm{O}_{2}$; d'autres auteurs (ForRester et al., 1966 ; PoskUTA, 1969 ; GALE \& TAKO, 1976) observent l'existence d'un effet inhibiteur de $\mathrm{O}_{2}$ qui se manifeste notamment lorsque la concentration en $\mathrm{O}_{2}$ est supérieure à celle de l'air ; à l'inverse, un léger effet stimulateur de $\mathrm{O}_{2}$ sur la photosynthèse du maïs a également été démontré (MOROT-GAudry et al., 1980 ; JoliveTTOURNIER, 1982).

Il apparaît donc pratiquement impossible de détecter une émission de gaz carbonique à la lumière par le maïs, ce qui laisserait supposer que ce végétal est exempt de photorespiration. La mise en cuvre de ${ }^{18} \mathrm{O}$ a cependant permis d'observer une photoconsommation d'oxygène par des feuilles excisées de maïs (VOLK \& JACKSON, 1972 ; FURBANK \& BADGER, 1982) ou par des plantes entières (ANDRE \& GERBAUD, 1979). Nous avons établi quelques propriétés de la photoconsommation d'oxygène de fragments foliaires de mais en examinant l'influence de certains facteurs: concentration en oxygène de la phase gazeuse, présence d'une forte teneur en gaz carbonique et âge du végétal. 


\section{MATÉRIEL ET MÉTHODES}

Le présent travail a été réalisé dans le Laboratoire de photosynthèse du Service de Radioagronomie au Centre d'Etudes Nucléaires de Cadarache.

\section{A. Matériel végétal}

Le mais (Zea mays L., variété «I.N.R.A. F7 $\times$ F2 » provenant du laboratoire de génétique et d'amélioration des plantes d'Estrées-Mons) est cultivé en pleine terre sous serre à toit cuvrant. Les expériences ont eu lieu au printemps. Le semis est périodique (12 pieds tous les $15 \mathrm{j}$., densité de 9 pieds $\cdot \mathrm{m}^{-2}$ ) et la date d'apparition des différentes feuilles sur chaque pied est notée. Ceci permet de disposer au moment de l'expérience de plants parvenus à des étapes de développement très variées (du stade plantule au stade floraison avancée) et de connaître l'âge des feuilles utilisées. Les jeunes feuilles ( 6 à 1.3 j.) correspondent à la feuille de rang 6 de plants âgés d'une trentaine de jours. Les autres échantillons proviennent des feuilles médianes $\left(6^{e}\right.$ à $8^{\mathrm{e}}$ feuille à partir de la base) de plants de 50 à $70 \mathrm{j}$.

Pour des expériences de comparaison avec un végétal de type métabolique $\mathrm{C}_{3}$, nous avons utilisé la tomate (Lycopersicum esculentum Mill., variété "Merveille des marchés ») ou le tabac (Nicotiana tabacum L., variété «JWB ") cultivés dans les mêmes conditions que le maïs.

Les folioles de tomate ont été prélevées sur des plants âgés de quelques semaines possédant 8 à 10 feuilles et ne présentant pas encore de ramification secondaire.

L'extraction de la chlorophylle se fait dans du méthanol pur et le dosage est effectué dans un mélange méthanol/eau $(90 / 10, v / v)$ par lecture des densités optiques à 645 et $663 \mathrm{~nm}$ (SCHMID, 1971)

\section{B. Dispositif expérimental}

Le montage utilisé pour les mesures de prise d'oxygène (fig. 1) est un circuit fermé (entrée d'air inférieure à 1 $\mathrm{\mu l} \cdot \mathrm{mn}^{-1}$ ) comprenant une chambre d'assimilation thermoréglée à $22^{\circ} \mathrm{C}$, destinée à recevoir l'échantillon végétal, une

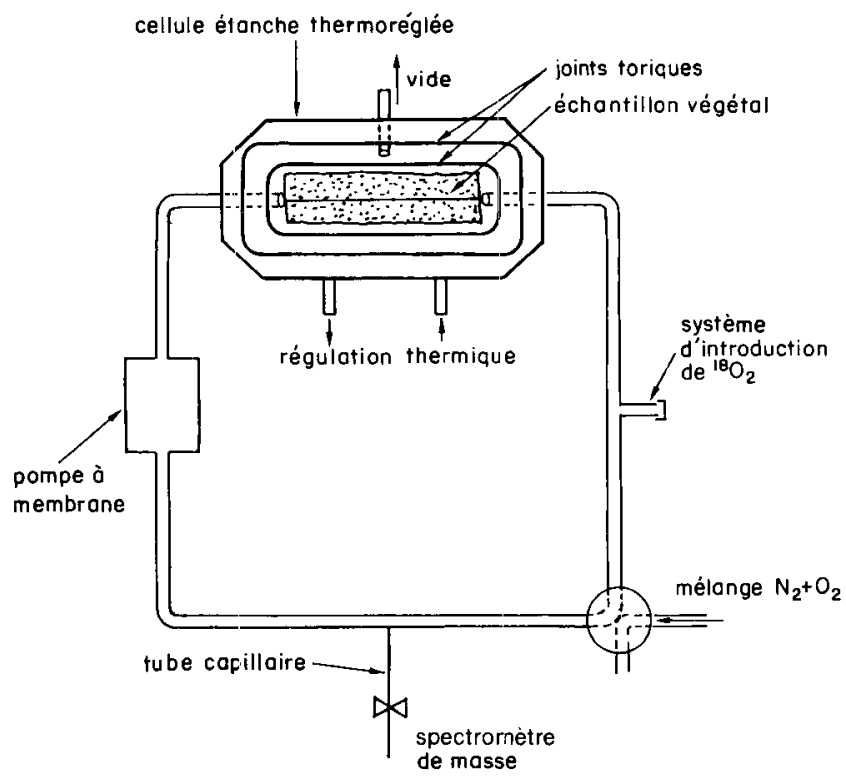

Figure 1

Schéma du montage utilisé dans les expériences de photoconsommation d'oxygène.

Schematic diagram of the apparatus used to measure rates of $\mathrm{O}_{2-}$ uptake. pompe à membrane (débit $240 \mathrm{I} \cdot \mathrm{h}^{-1}$ ) assurant une homogénéisation rapide de la phase gazeuse dans le circuit et un système d'introduction de gaz. Le circuit dont le volume est voisin de $75 \mathrm{ml}$ est relié par un tube capillaire à la source d'ions d'un spectromètre de masse (ATLAS $\mathrm{CH}_{4}$ ).

\section{Protocole opératoire}

L'échantillon foliaire, environ $1 \mathrm{~g}$. de matière fraîche (MF), est balayé à l'obscurité en circuit ouvert pendant $5 \mathrm{mn}$ par un mélange d'azote et d'oxygène dont les concentrations sont connues. Le montage est ensuite fermé sur luimême et $1 \mathrm{ml}$ d'oxygène, enrichi à $95 \mathrm{p} .100$ en ${ }^{18} \mathrm{O}$ (Bureau des Isotopes Stables, CEN Saclay), est introduit. Le végétal est soumis à des alternances obscurité-lumière ; l'éclairement (lampe à filament de tungstène à faisceau froid MAZDAPAR «F»150 W) est fixé à $800 \mu \mathrm{E} \cdot \mathrm{m}^{-2} \cdot \mathrm{s}^{-1}$ entre 400 et $700 \mathrm{~nm}$. Un volume connu de gaz carbonique (1 à $2 \mathrm{ml}$ ) est, dans certains cas, injecté dans le circuit à la lumière afin de mesurer la capacité photosynthétique du végétal à concentration saturante en $\mathrm{CO}_{2}$.

\section{Analyse isotopique de la phase gazeuse}

Les spectres de masse de l'atmosphère du circuit sont obtenus à intervalles de temps réguliers. Les intensités des pics de masse de même que le temps correspondant à chaque balayage sont automatiquement transcrits sur une imprimante.

La teneur en ${ }^{17} \mathrm{O}$ de l'oxygène enrichi en ${ }^{18} \mathrm{O}$ utilisé étant inférieure à $0,7 \mathrm{p} .100$, la contribution des espèces moléculaires ${ }^{16} \mathrm{O}{ }^{17} \mathrm{O},{ }^{17} \mathrm{O}{ }^{17} \mathrm{O}$ et ${ }^{18} \mathrm{O}{ }^{17} \mathrm{O}$ est négligée. D'autre part, la teneur en ${ }^{18} \mathrm{O}$ étant très élevée, la probabilité d'existence de ${ }^{16} \mathrm{O}{ }^{18} \mathrm{O}$ est faible devant celle de ${ }^{18} \mathrm{O}{ }^{18} \mathrm{O}$ si bien que seuls les pics de masse $32\left({ }^{16} \mathrm{O}{ }^{16} \mathrm{O}\right)$ et $36\left({ }^{18} \mathrm{O}{ }^{18} \mathrm{O}\right)$ sont pris en considération. Pour le gaz carbonique, seul le pic 44 correspondant à l'espèce ${ }^{12} \mathrm{C}^{16} \mathrm{O}_{2}$ a été retenu ; il n'a pas été tenu compte des autres pics de masse qui mettent en jeu, à leur concentration naturelle, les isotopes ${ }^{13} \mathrm{C}$ $(1,1$ p. 100$),{ }^{17} \mathrm{O}(0,037$ p. 100$)$ et ${ }^{18} \mathrm{O}(0,2$ p. 100$)$. L'argon (pic de masse 40, concentration naturelle dans l'air 0,934 p. 100) est utilisé comme référence interne : la vitesse de disparition de l'argon de la phase gazeuse du circuit reflète la consommation propre du spectromètre de masse.

\section{RÉSULTATS}

\section{A. Vitesses de prise d'oxygène et de gaz carbonique par le mais : comparaison avec la tomate}

Les variations en fonction du temps des concentrations en $\mathrm{O}_{2}$ et $\mathrm{CO}_{2}$ dans la phase gazeuse surmontant un échantillon foliaire de maïs, lors d'une expérience type, sont représentées sur la figure $2 \mathrm{~A}$. Lorsque le végétal est à l'obscurité, on observe une prise d'oxygène et un dégagement concomitant de gaz carbonique qui mesurent la respiration à l'obscurité. A la lumière, la concentration en $\mathrm{CO}_{2}$ diminue et atteint, à l'issue de quelques minutes, le point de compensation voisin de $0 \mu \mathrm{l} \cdot \mathrm{l}^{-1}$. Parallèlement, la concentration en $\mathrm{O}_{2}$ augmente et se stabilise. Si l'on enrichit alors l'atmosphère en $\mathrm{CO}_{2}$ (concentration initiale voisine de 4 p. 100), on constate de nouveau une prise de $\mathrm{CO}_{2}$ et le dégagement de $\mathrm{O}_{2}$ qui lui correspond.

Les variations relatives aux 2 espèces isotopiques de l'oxygène, 32 et 36 , sont représentées sur la figure $2 \mathrm{~B}$. Pendant les périodes d'obscurité, ${ }^{16} \mathrm{O}_{2}$ et ${ }^{18} \mathrm{O}_{2}$ disparaissent 
ensemble ; par contre, à la lumière, la concentration en ${ }^{16} \mathrm{O}_{2}$ augmente tandis que celle en ${ }^{18} \mathrm{O}_{2}$ diminue et ce, plus rapidement qu'à l'obscurité. Il a été vérifié que la lumière ne crée pas au sein du végétal des conditions favorables à un échange isotopique entre l'oxygène moléculaire et l'eau intracellulaire et que l'augmentation de la vitesse de disparition de l'espèce ${ }^{18} \mathrm{O}_{2}$ est uniquement due à une stimulation, sous lumière, de la consommation d'oxygène par le végétal (Dimon, 1977).

$\mathrm{La}$ variation en fonction du temps du volume de ${ }^{18} \mathrm{O}_{2}\left(\mathrm{~V}_{36}\right)$ dans le montage est représentée par une succession de droites en coordonnées semi-logarithmiques (fig. 2B). La pente de chaque segment de droite est une mesure de la constante de vitesse, $\mathrm{k}$, relative à la prise de l'espèce 36 pour la phase du traitement correspondant.

Soit :

$$
\frac{\mathrm{dV}_{36}}{\mathrm{dt}}=-\mathrm{kV}_{36}
$$
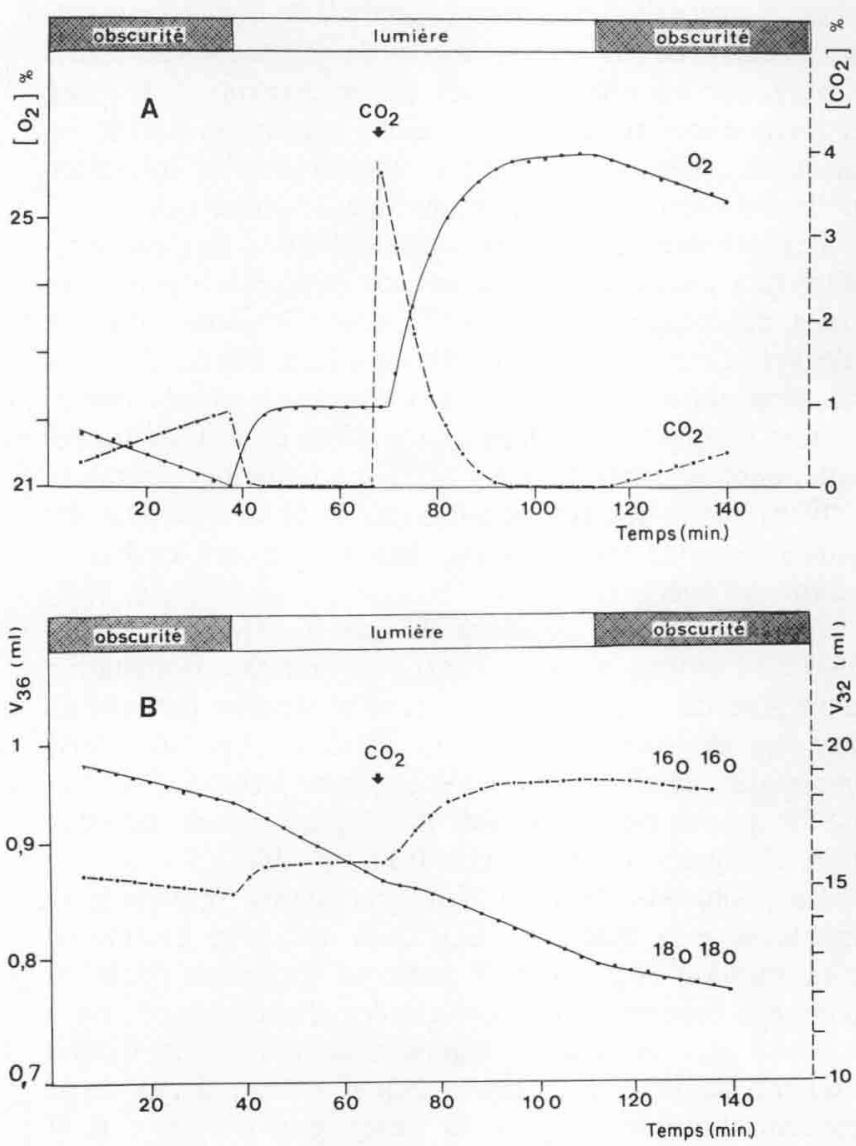

Figure 2

Evolution au cours du temps des concentrations en $\mathrm{O}_{2}$ et $\mathrm{CO}_{2}$ de la phase gazeuse $(A)$ et des volumes relatifs aux espèces isotopiques de l'oxygène (B): ${ }^{16} \mathrm{O}{ }^{16} \mathrm{O}$ (32) et ${ }^{18} \mathrm{O}^{18} \mathrm{O}$ (36). Maïs, 800 $\mu E \cdot m^{-2} \cdot s^{-1}, 22^{\circ} \mathrm{C}$.

Variation with time of $\mathrm{O}_{2}$ and $\mathrm{CO}_{2}$ concentrations in the atmosphere (A) and of the different isotopic species $(B):{ }^{16} \mathrm{O}^{16} \mathrm{O}(32),{ }^{18} \mathrm{O}^{18} \mathrm{O}$ (36). Maize, $800 \mu E \cdot m^{-2} \cdot \mathrm{s}^{-1}, 22^{\circ} \mathrm{C}$.

Si on néglige les éventuels effets isotopiques dus à la différence des masses 16 et 18 , la consommation de $\mathrm{O}_{2}$ à la lumière se calcule à partir de la relation :

$$
\frac{\mathrm{dV}_{\mathrm{O}_{2}}}{\mathrm{dt}}=-\mathrm{k}\left(\mathrm{V}_{36}+\mathrm{V}_{32}\right)
$$

Comme les expériences sont conduites en circuit fermé, la prise d'oxygène à la lumière $(\mathrm{RL})$ est mesurée au point de compensation en $\mathrm{CO}_{2}$. L'intensité de la photosynthèse à concentration saturante en $\mathrm{CO}_{2}$ est évaluée par la vitesse initiale de disparition de l'espèce 44 dans la phase gazeuse après injection de $\mathrm{CO}_{2}$ dans le montage $(\mathrm{P})$. L'intensité de la respiration à l'obscurité ( $\mathrm{RO})$ est déterminée en mesurant les vitesses de disparition de $\mathrm{O}_{2}\left({ }^{18} \mathrm{O}_{2}\right.$ ou $\left.{ }^{16} \mathrm{O}_{2}\right)$ ou d'apparition de $\mathrm{CO}_{2}$.

Les résultats d'expériences conduites sur des feuilles adultes de maïs ( 30 à $40 \mathrm{j}$.) sont réunis dans le tableau 1 . Ils sont rapportés à la surface foliaire ou à la teneur en chlorophylle de l'échantillon végétal. Pour comparaison, des expériences identiques ont été faites sur des folioles de tomate.

\section{TABLEAU 1}

Vitesses de prise d'oxygène à l'obscurité (RO) et à la lumière au point de compensation en $\mathrm{CO}_{2}(R L)$ mesurées sur des feuilles adultes de maïs et des folioles de tomate. $P$ représente la vitesse de consommation de $\mathrm{CO}_{2}$ à forte teneur en $\mathrm{CO}_{2}(4 \mathrm{p} .100)$. Les valeurs ont été obtenues à partir de 10 expériences dans le cas du maïs, de 4 dans le cas de la tomate.

Rates of $\mathrm{O}_{2}$-uptake in the dark (RO) and in the light at the $\mathrm{CO}_{2}$ compensation point ( $R L)$ on mature leaves of maize and tomato. $P$ is the rate of $\mathrm{CO}_{2}$-uptake at high $\mathrm{CO}_{2}$ concentration (4\%). Values were

\begin{tabular}{|c|c|c|c|c|}
\hline & \multicolumn{2}{|c|}{$\mu \mathrm{mol} . \mathrm{s}^{-1} \cdot \mathrm{m}^{-2}$} & \multicolumn{2}{|c|}{$\mathrm{m} \mathrm{mol} \cdot \mathrm{s}^{-1} \cdot \mathrm{mol}^{-1} \mathrm{Chl}$} \\
\hline & Maïs & Tomate & Maïs & Tomate \\
\hline RO & $0,8 \pm 0,1$ & $0,7 \pm 0,3$ & $1,0 \pm 0,2$ & $1,3 \pm 0,7$ \\
\hline RL & $3,0 \pm 0,4$ & $10,9 \pm 1,7$ & $3,9 \pm 0,7$ & $25,4 \pm 5,1$ \\
\hline $\mathbf{P}$ & $16,4 \pm 2,4$ & $15,6 \pm 1,5$ & $21,3 \pm 6,1$ & $38,3 \pm 8,6$ \\
\hline
\end{tabular}
obtained from 10 experiments with maize, 4 with tomato.

Il apparaît chez le maïs une stimulation de la prise d'oxygène par la lumière. La photoconsommation d'oxygène représente environ 4 fois la respiration à l'obscurité ; elle est cependant plus faible que celle qui est observéc chez la tomate ou d'autre végétaux de type $\mathrm{C}_{3}$ :

tabac, $\mathrm{RL}=60,8 \mu \mathrm{mol} \cdot \mathrm{s}^{-1} \cdot \mathrm{kg}^{-1} \mathrm{MF}$;

soja, $\mathrm{RL}=47,2 \pm 13,9 \mu \mathrm{mol} \cdot \mathrm{s}^{-1} \cdot \mathrm{kg}^{-1} \mathrm{MF} \quad$ (DIMON, 1977).

\section{B. Influence de la concentration en oxygène et d'une forte teneur en gaz carbonique}

Les variations des vitesses de prise de $\mathrm{O}_{2}$ à la lumière et à l'obscurité en fonction de la concentration en $\mathrm{O}_{2}$ de la phase gazeuse apparaissent sur la figure 3 . La respiration à l'obscurité est pratiquement saturée pour une concentration en $\mathrm{O}_{2}$ voisine de $3 \mathrm{p} .100$ alors que la photoconsommation de $\mathrm{O}_{2}$ augmente de manière continue entre 2 et 21 p. 100 : à 21 p. 100 de $\mathrm{O}_{2}$, elle est double de celle observée à 2 p. 100.

Lorsque du gaz carbonique est injecté à la lumière dans le circuit (teneur initiale 4 p. 100), l'activité photosynthétique ramène progressivement la concentration de $\mathrm{CO}_{2}$ à celle du point de compensation voisin de $0 \mu \mathrm{l} \cdot \mathrm{I}^{-1}$ (fig. 2). Parallèlement, on remarque que la consommation de ${ }^{18} \mathrm{O}_{2}$ diminue beaucoup, voire s'annule dans certains cas, dans le domaine des très fortes concentrations en $\mathrm{CO}_{2}$ (entre 4 et 2 p. 100). On note qu'elle s'accélère de nouveau bien avant que le point de compensation en $\mathrm{CO}_{2}$ ne soit atteint. 


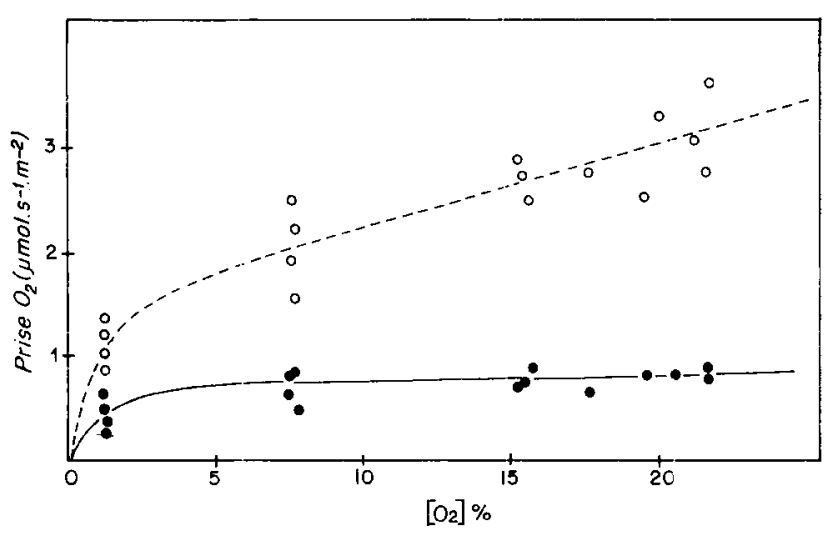

Figure 3

Influence de la teneur en $O_{2}$ de l'atmosphère sur la prise de $O_{2}$. Les courbes ont été tracées à partir des données de 4 expériences, pour chaque concentration en $\mathrm{O}_{2}$, conduites sur des feuilles de maïs âgées de $25 \grave{a} 34 j$

$\circ R L$

$R O$

Effect of $\mathrm{O}_{2}$ concentration on the rate of $\mathrm{O}_{2}$-uptake. For each $\mathrm{O}_{2}$ concentration, 4 experiments were carried out with mature leaves of maize ( 25 to 34 days).

$\bigcirc R L \bullet R O$

\section{Influence de l'âge des feuilles sur la prise d'oxygène}

Dans ces expériences, les vitesses de prise d'oxygène à la lumière et à l'obscurité sont mesurées dans une atmosphère de $\mathrm{N}_{2}+21$ p. $100 \mathrm{O}_{2}$. La figure $4 \mathrm{~A}$. représente les variations, en fonction de l'âge de la feuille, des vitesses de consommation d'oxygène rapportées à la surface de l'échantillon foliaire.

Alors que la respiration à l'obscurité paraît indépendante de l'âge des feuilles, la photoconsommation d'oxygène

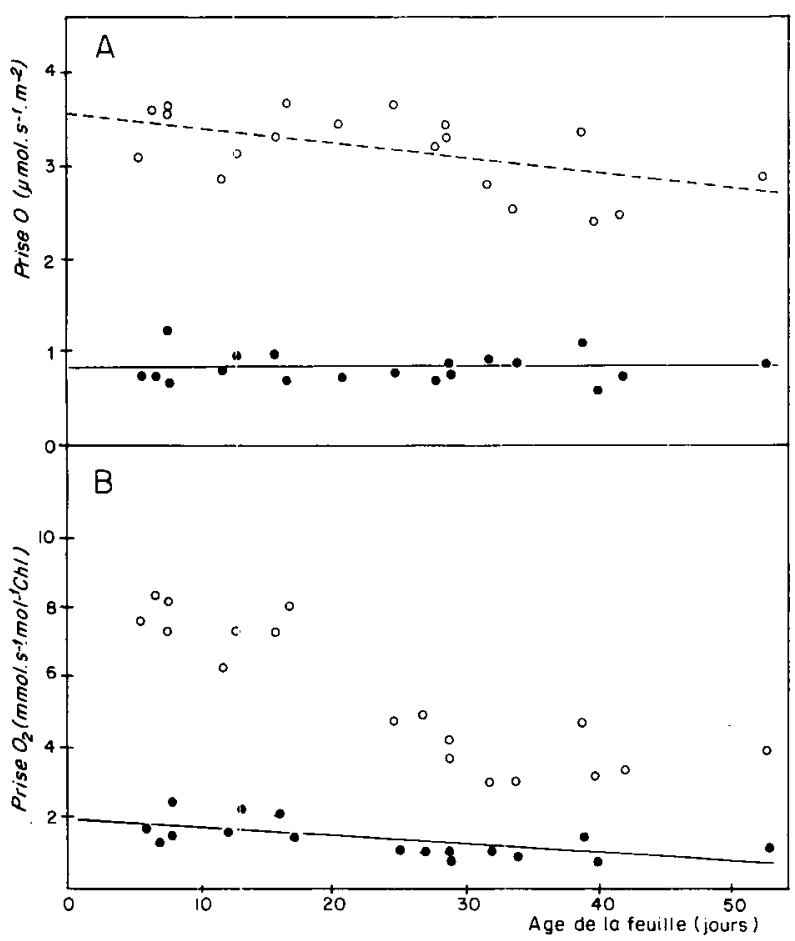

Figure 4

Influence de l'âge de la feuille sur la prise d'oxygène rapportée à la surface de l'échantillon foliaire $(A)$ ou à la teneur en chlorophylle $(B)$.

$\bigcirc R L$

Effect of plant development on $\mathrm{O}_{2}$-uptake in relation to leaf area $(A)$ or chlorophyll content $(B)$.

$\circ R L$

- $\mathrm{KO}$ semble diminuer légèrement lorsque l'âge de la feuille augmente. L'analyse de variance effectuée sur 2 groupes de feuilles de classes d'âge différentes indique que cette diminution est significative au seuil de 1 p. 100 . L'effet apparait plus nettement lorsque les valeurs de prise d'oxygène sont rapportées à la teneur en chlorophylle (Chl) des échantillons (fig. 4B). Les photoconsommations d'oxygène selon les populations envisagées sont les suivantes :

- $\mathrm{RL}=7,6 \pm 0,7 \mathrm{mmol} \cdot \mathrm{s}^{-1} \cdot \mathrm{mol}^{-1} \mathrm{Chl}$ pour les feuilles jeunes (moins de $20 \mathrm{j}$ ) ;

- $\mathrm{RL}=3,9 \pm 0,7 \mathrm{mmol} \cdot \mathrm{s}^{-1} \cdot \mathrm{mol}^{-1} \mathrm{Chl}$ pour les feuilles adultes et âgées (plus de $20 \mathrm{j}$ ).

\section{DISCUSSION ET CONCLUSION}

L'utilisation de l'isotope est la seule façon d'accéder directement à la consommation d'oxygène d'un végétal. Les vitcsses calculées constituent cependant des estimations par défaut de la vitesse réclle de photoconsommation d'oxygène; en effet, les sites de production et de prise d'oxygène sont voisins dans la cellule végétale et il n'est pas facile de prendre en compte une éventuelle utilisation préférentielle in situ de l'oxygène photosynthétique.

Les résultats obtenus concernant l'étude des échanges d'oxygène chez le maïs montrent que ce végétal présente, au point de compensation en $\mathrm{CO}_{2}$, une photoconsommation d'oxygène environ 4 fois plus élevée à 21 p. 100 de $\mathrm{O}_{2}$ que la consommation à l'obscurité. Les valeurs de photoconsommation d'oxygène obtenues sont voisines de celles estimées antérieurement par Dimon (1977) sur le même végétal.

Nous avons observé en outre que la prise d'oxygène est plus importante chez les jeunes feuilles que chez les feuilles adultes et âgées.

Comme chez les végétaux de type $\mathrm{C}_{3}$ (Mulchi et al., 1971; CANVIN et al., 1980), la photoconsommation d'oxygène du maïs augmente quand la pression partielle en oxygène de l'atmosphère croît de 2 à 21 p. 100 . Cette observation confirme et complète celle de VOLK \& JACKSON (1972) qui ont montré, à l'aide de ${ }^{18} \mathrm{O}$, une stimulation de la prise d'oxygène du maïs entre 0 et 8 p. 100 .

La photoconsommation d'oxygène paraît pratiquement insensible à la teneur en $\mathrm{CO}_{2}$ dans une large gamme de concentration ( 1 p. 100 à 0$)$; elle est fortement diminuée pour des concentrations plus élevées. Parallèlement, on a constaté que la prise d'oxygène à la lumière de feuilles excisées de mais était pratiquement indépendante de la concentration en $\mathrm{CO}_{2}$ de la phase gazeuse entre 0 et $400 \mu \mathrm{l} \cdot \mathrm{l}^{-1}$ (FURBANK \& BADGER, 1982). ANDRE \& GERBAUD (1979) ont trouvé, sur plants de maïs entiers, que la photoconsommation d'oxygène demeure pratiquement inchangée entre 300 et $600 \mu \mathrm{l} \cdot 1^{-1}$ de $\mathrm{CO}_{2}$; par contre, quand le $\mathrm{CO}_{2}$ devient limitant, il apparaît une photoconsommation d'oxygène supplémentaire tandis que la photosynthèse et la respiration diminuent.

L'acide glycolique apparaît comme le métabolite clef de la photorespiration; sa synthèse par oxydation d'intermédiaires du cycle de CALVIN (ribulose-1,5-bisphosphate ou oses monophosphorylés) permettrait d'expliquer en grande partie la photoconsommation d'oxygène observée; son métabolisme ultérieur conduirait à un dégagement de gaz carbonique. Pour CANVIN (1979), la stimulation par la lumière de la prise de ${ }^{18} \mathrm{O}_{2}$ observée chez les végétaux de type $\mathrm{C}_{4}$ ne serait pas associée à une biosynthèse de glycolate mais serait due principalement à une réduction de l'oxygène 
atmosphérique par l'appareil photosynthétique (réaction de type MEHLER, 1951). Il est vrai qu'il n'est pas possible, avec ${ }^{18} \mathrm{O}$, de faire la part entre ces différents processus. Cependant, les organites et les enzymes intervenant dans le métabolisme du glycolate sont présents chez les plantes de type $\mathrm{C}_{4}$ et se trouvent localisés dans une structure cellulaire particulière: le parenchyme périvasculaire (OSMOND \& HARRIS, 1971 ; WAGHMODE \& JoSHI, 1982) dont les cellules isolées présentent au surplus un effet WARBURG (CHOLLET \& OGREN, 1972). L'existence du métabolisme du glycolate a été établi chez des fragments foliaires de maïs : des expériences d'incorporation de ${ }^{14} \mathrm{CO}_{2}$ en photosynthèse stationnaire se traduisent par un marquage de 2 dérivés du glycolate, la glycine et la sérine, marquage sensible à la concentration en oxygène de l'atmosphère ainsi qu'aux inhibiteurs usuels de la voie du glycolate (MAHON et al., 1974 ; LEWANTY \& MALESZEWSKI, 1976 ; MOROT-GAUdRY et al., 1980). D'autre part, nous avons bien établi que la photoconsommation de ${ }^{18} \mathrm{O}_{2}$ s'accompagne, chez le mais, tout comme chez les végétaux de type $C_{3}$, d'une incorporation de l'isotope dans la glycine et la sérine (Dimon et al., 1977). Enfin, nous avons montré que les caractéristiques des échanges d'oxygène sont très comparables à celles rencontrées chez les plantes de type $\mathrm{C}_{3}$.

Par conséquent, il ne semble pas y avoir de raison fondamentale pour dénier l'existence chez le maïs d'un métabolisme photorespiratoire au sens strict. Pour expliquer que ce phénomène se manifeste de façon relativement peu intense, on peut invoquer 2 hypothèses : d'une part, il y aurait un effet de concentration dans les cellules du parenchyme périvasculaire du gaz carbonique provenant des acides dicarboxyliques, ce qui réduirait l'activité oxygénasique de la ribulose-1,5-bisphosphate carboxylase/oxygénase présente dans ces tissus (BERRY \& FARQUHAR, 1977); d'autre part, l'émission de gaz carbonique photorespiratoire serait masquée du fait d'une reprise très active de ce gaz par la phosphoénolpyruvate carboxylase présente dans le mésophylle (El-SharKaWy et al., 1967; HEW et al., 1969 ; RATHNAM, 1979). Les critères habituels de mesure de la capacité photorespiratoire basés sur les seuls échanges de $\mathrm{CO}_{2}$ ne paraissent donc pas applicables aux végétaux de type $\mathrm{C}_{4}$.

Reçu le 20 janvier 1983. Accepté le 18 mai 1983.

\section{RÉFÉRENCES BIBLIOGRAPHIQUES}

Andre M., Gerbaud A., 1979. Consommation d'oxygène pendant la photosynthèse chez Zea mays. C.R. Acad. Sci., Paris, 289, 793-796. Berry J., Farquhar G., 1977. The $\mathrm{CO}_{2}$ concentrating function of $\mathrm{C}_{4}$ photosynthesis. A biochemical model. p. 119-131. In D. O. Hall, J. Coombs \& T. W. Goodwin, Proc. 4th Int. Congr. Photosynthesis. Biochemical Society, London.

Canyin D. T., 1979. Photorespiration : comparison between $\mathrm{C}_{3}$ and $\mathrm{C}_{4}$ plants p. 368-396. In M. Gibbs \& E. Latzko. Encyclopedia of Plant Physiology. Vol. 6, Photosynthesis II, Springer Verlag, Berlin, Heidelberg, New York.

Canvin D. T., Berry J. A., Badger M. R., Fock H., Osmond C. B., 1980. Oxygen exchange in leaves in the light. Plant Physiol., 66, 302-307.

Chollet R., Ogren W. L., 1972. Oxygen inhibits maize bundle sheath photosynthesis. Biochem. Biophys. Res. Commun., 46, 2062-2066.

Dimon B., 1977. Contribution à l'étude du métabolisme de l'oxygène au cours de la photorespiration. Thèse de doctorat d'Etat. Académie de Montpellier. Université des Sciences et Techniques du Languedoc. $118 \mathrm{p}$.

Dimon B., Gerster R., Tournier P., 1977. Photoconsommation d'oxygène et biosynthèse de la glycine et de la sérine chez Zea mays. C.R. Acad. Sci., Paris, 284, 297-299.

El-Sharkawy M. A., Loomis R. S., Williams W. A., 1967. Apparent reassimilation of respiratory carbon dioxide by different plant species. Physiol. Plant., 20, 171-186.

Forrester M. L., Krotkov G., Nelson C. D., 1966. Effect of oxygen on photosynthesis, photorespiration and respiration in detached leaves. II. Corn and other monocotyledons. Plant Physiol., 41, 428431.

Furbank R. T., Badger M. R., 1982. Photosynthetic oxygen exchange in attached leaves of $\mathrm{C}_{4}$ monocotyledons. Aust. J. Plant Physiol., 9, 553-558.

Gale J., Tako T., 1976. Response of Zea mays and Phaseolus vulgaris to supra-atmospheric concentrations of oxygen. Photosynthetica, 10, 89-92.

Hew C. S., Krotkov G., Canvin D. T., 1969. Determination of the rate of $\mathrm{CO}_{2}$ evolution by green leaves in light. Plant Physiol., 44, $662-670$.

Jolivet-Tournier P., 1982. Contribution à l'étude du métabolisme de l'oxygène dans la photorespiration. Exemple du maïs. Thèse de Doctorat d'Etat. Université d'Aix-Marseille II. Faculté des Sciences de Luminy. $125 \mathrm{p}$.
Lewanty Z., Maleszewski S., 1976. Conversion of photosynthetic products in the light in $\mathrm{CO}_{2}$-free $\mathrm{O}_{2}$ and $\mathrm{N}_{2}$ in leaves of Zea mays $\mathrm{L}$. and Phaseolus vulgaris L. Planta, 131, 121-123.

Mahon J. D., Fock H., Höhler T., Canvin D. T., 1974. Changes in specific radioactivities of corn-leaf metabolites during photosynthesis in ${ }^{1+} \mathrm{CO}_{2}$ and ${ }^{12} \mathrm{CO}_{2}$ at normal and low oxygen. Planta, 120, 113123.

Mehler A. H., 1951. Studies on reactions of illuminated chloroplasts. I. Mechanism of the reduction of oxygen and other Hill reagents. Arch. Biochem. Biophys., 33, 65-77.

Morot-Gaudry J. F., Farineau J. P., Huet J. C., 1980. Oxygen effect on photosynthetic and glycolate pathways in young maize leaves. Plant Physiol., 66, 1079-1084.

Mulchi C. L., Volk R. J., Jackson W. A., 1971. Oxygen exchange of illuminated leaves at carbon dioxide compensation, p. 35-50. In H. D. Hatch, C. B. Osmond \& R. O. Slatyer, Photosynthesis and Photorespiration, Wiley-Interscience.

Osmond C. B., Harris B., 1971. Photorespiration during $\mathrm{C}_{4}$ photosynthesis. Biochim. Biophys. Acta, 234, 270-282.

Poskuta J., 1969. Photosynthesis, respiration and post-illumination fixation of $\mathrm{CO}_{2}$ by corn leaves as influenced by light and oxygen concentration. Physiol. Plant., 22, 76-85.

Rathnam C. K. M., 1979. Metabolic regulation of carbon flux during $\mathrm{C}_{4}$ photosynthesis. II. In situ evidence for refixation of photorespiratory $\mathrm{CO}_{2}$ by $\mathrm{C}_{4}$ phosphoenolpyruvate carboxylase. Planta, 145, 13-23.

Schmid G. H., 1971. Origin and properties of mutant plants yellow tobacco. in Methods in Enzymology, XXIII, part A, 171194.

Volk R. J., Jackson W. A., 1972. Photorespiratory phenomena in maize, oxygen uptake, isotope discrimination and carbon dioxide efflux. Plant Physiol., 49, 218-223.

Voskresenskaya N. P., Polyakov M. A., Karpushkin L. T., 1974 Effect of oxygen concentration on carbon dioxide exchange in beans and corn. Sov. Plant Physiol, 21, 367-372.

Waghmode A. P., Joshi G. V., 1982. Photosynthetic and photorespiratory enzymes and metabolism of ${ }^{1+} \mathrm{C}$-substrates in isolated leaf cells of the $\mathrm{C}_{4}$ species Aeluropus lagopoides L. Photosynthetica, 16, 17-21.

Zelitch I., 1971. Photosynthesis, photorespiration and plant productivity. Academic press, New York and London, $347 \mathrm{p}$. 